\title{
CONFLICTOS ENTRE DERECHOS FUNDAMENTALES. UNA CRÍTICA A LUIGI FERRAJOLI *
}

\section{Giorgio Pino}

Universidad de Palermo

RESUMEN. El objeto de este breve texto es poner de relieve alguna duda acerca de un específico, aunque no marginal, aspecto de la compleja teoría de los derechos fundamentales construida por FERRAJOLI: el problema del conflicto entre derechos fundamentales. En primer lugar, ofreceré un sumario bastante genérico sobre la presencia (por lo menos aparente, o potencial) de conflictos entre derechos fundamentales en el panorama constitucional contemporáneo. De ahí pasaré a ilustrar la estrategia elaborada por L. FERRAJOLI para argumentar la tesis de la ausencia o, al menos, la extremadamente reducida y marginal presencia de los conflictos entre derechos fundamentales. Por último, mostraré algunas posibles críticas a la estrategia de FERRAJOLI.

Palabras clave: L. FERRAJOLI, derechos fundamentales, conflictos entre derechos.

ABSTRACT. This essay deals with a specific — but by no means marginal- feature of the complex and rich theory of fundamental rights developed by L. FERRAJOLI, the problem of conflicts of rights. To begin with, I will draw a quite generic theoretical framework about (at least prima facie, or potential) conflicts between fundamental rights in the context of contemporary constitutional states. Then I will describe L. FERRAJOL's thesis about the absence, or at least the very marginal role of conflicts between fundamental rights. Conclusively, I will point to some possible critiques to the strategy elaborated by FERRAJOLI in order to reduce the impact of conflicts among fundamental rights.

Keywords: L. FERRAJOLI, fundamental rights, conflicts of rights.

* Fecha de recepción: 28 de septiembre de 2009. Fecha de aceptación: 29 de octubre de 2009. 
FerRAJoli ha elaborado, en los últimos diez años, una teoría de los derechos fundamentales rica, compleja y perfectamente integrada en una más general teoría del Derecho y de la democracia que ha estado elaborando al mismo tiempo ${ }^{1}$. La teoría de los derechos propuesta por FERRAJOLI contiene una multiplicidad de aspectos dignos de atención, tanto desde un punto de vista de la filosofía del Derecho y de la filosofía política (por ejemplo, las relaciones entre la teoría de los derechos y la igualdad y la democracia constitucional) como desde un punto de vista de propuestas más específicas, con frecuencia ciertamente originales, de análisis teórico y de pulcritud conceptual del lenguaje de los derechos (pienso, por ejemplo, en la propuesta de una definición puramente formal de Derecho fundamental, o en la distinción entre garantías primarías y garantías secundarias ${ }^{2}$ ).

Muchos de estos aspectos filosóficos y teóricos de la teoría de los derechos fundamentales de FerRAJOLI han sido ya ampliamente debatidos ${ }^{3}$. En este breve texto pretendo poner de relieve alguna duda acerca de un específico, aunque no marginal, aspecto de la compleja teoría de los derechos fundamentales construida por FERRAJOLI: el problema del conflicto entre derechos fundamentales. De hecho, creo que la manera en que FERRAJOLI aborda este específico problema, si he comprendido bien, presenta algunos puntos débiles — puntos que, de enmendarse, quizás podrían enriquecer, en conjunto, el potencial explicativo de su sofisticada teoría.

En primer lugar, ofreceré un sumario bastante genérico sobre la presencia (por lo menos aparente, o potencial) de conflictos entre derechos fundamentales en el panorama constitucional contemporáneo $(\mathbb{1}$ ). De ahí pasaré a ilustrar la estrategia elaborada por L. FERRAJOLI para argumentar la tesis de la ausencia o, al menos, la extremadamente reducida y marginal presencia de los conflictos entre derechos fundamentales $(\mathbb{S}$ 2.1-2.2). Por último, mostraré algunas posibles críticas a la estrategia de FERRAJOLI $(\mathbb{S} 3$ y 4$)$.

${ }^{1}$ L. Ferrajoli, Diritti fondamentali, en L. FerRajoli, Diritti fondamentali. Un dibattito teorico, a cura di E. ViTALE, Roma-Bari, Laterza, 2001, pp. 5-40; I diritti fondamentali nella teoria del diritto, ivi; I fondamenti dei diritti fondamentali, ivi, pp. 277-369; «Per un costituzionalismo di diritto privato», Rivista critica del diritto privato, 1, 2004; «Diritti fondamentali e democrazia costituzionale», Analisi e diritto, 2002-2003, pp. 331-350; «Diritto civile e principio di legalità», Europa e diritto privato, 3, 2005; «Le garanzie costituzionali dei diritti fondamentali», Teoria politica, 1, 2007; Principia iuris, 2 vols., RomaBari, Laterza, 2007; vid. también A. GarCía FigueroA, «Entrevista a L. Ferrajoli», en M. CARBOnell y P. Salazar (coords.), Garantismo. Estudios sobre el pensamiento jurídico de L. Ferrajoli, Madrid, Trotta, 2005, pp. 515-536.

2 L. FerRAJOLI, Diritti fondamentali, cit.; Id., Principia iuris, vol. 1, cit., pp. 196-197, 668-695 (especialmente p. 673) califica como «garantía primaria» de un derecho la prestación exigida para satisfacer el contenido del mismo derecho (y, por ello, lógicamente implicada). Las «garantías secundarias» de un derecho subjetivo son, en cambio, el conjunto de los mecanismos institucionales necesarios para asegurar el disfrute y la tutela efectiva del Derecho, $c f r$. L. FERRAJOLI, Diritti fondamentali, cit., pp. 26-33; Id., Principia iuris, vol. 1, cit., pp. 675-679.

${ }^{3}$ Cfr. en particular las contribuciones de R. Guastini, M. Jori y A. PinTORE en Diritti fondamentali. Un dibattito teorico, cit.; F. POGGI, «Diritti d'agire, permessi e garanzie», Analisi e diritto, 2002-2003, pp. 241-274. Para una discusión sobre las construcciones teóricas generales de FERRAJOLI, incluida la parte de los derechos, M. CARBonell y P. SAlazAR (coords.), Garantismo, cit. 


\section{EL PROBLEMA DEL CONFLICTO ENTRE DERECHOS FUNDAMENTALES EN EL PANORAMA CONSTITUCIONAL CONTEMPORÁNEO}

El panorama constitucional contemporáneo (las cartas constitucionales de los países democráticos, y la práctica de su interpretación y aplicación por parte de las respectivas jurisdicciones constitucionales y ordinarias) exhibe algunos rasgos peculiares, y, en ocasiones, incluso paradójicos. Las constituciones contemporáneas declaran, en efecto, muchos y heterogéneos derechos fundamentales (son constituciones «largas»), expresados en términos bastante amplios e indeterminados que hacen necesario operaciones de especificación y concretización. Además, los derechos son a menudo incompatibles entre ellos, en raras ocasiones o nunca están ordenados por relaciones jerárquicas o de prioridad, sino que - más bien al contrario- están expresamente dispuestos en un plano (por lo menos formalmente) de paridad o equidad. Es cierto que, en ocasiones, la proclamación de un derecho fundamental va acompañada de la previsión de posibles derogaciones, lo que sustancialmente equivale a indicar un orden de prioridad entre el Derecho «derogado» y aquello que lo deroga (para la constitución italiana, por ejemplo, la «buena costumbre» es un límite expreso a la libertad de expresión y a la libertad religiosa; la igualdad, la dignidad personal y la utilidad social son límites expresos respecto de las libertades económicas y de mercado). Sin embargo, estas derogaciones son a su vez formuladas en términos tan amplios como las mismas formulaciones de los derechos y, por tanto, necesitan a su vez ser concretizadas, especificadas, etc.

Además, mientras que muchos derechos constitucionales son expresamente calificados como «inviolables», en la práctica del tratamiento jurisdiccional y legislativo de los derechos fundamentales, en cambio, es considerada una cuestión pacífica que los derechos fundamentales sean «razonablemente» limitados, incluso más allá de los límites eventualmente indicados en el texto constitucional de manera específica para cada Derecho. Los límites a los derechos fundamentales son normalmente individuados, ya sea por vía legislativa o por vía interpretativa, para evitar que la tutela de un derecho interfiera excesivamente en otros derechos fundamentales o, incluso, con exigencias diferentes respecto a los derechos fundamentales, como ciertos intereses públicos o colectivos; o para individuar un nivel de tutela de los derechos compatibles con los recursos financieros y materiales disponibles, etcétera.

Explicado de modo extremadamente sintético, el rasgo característico de las constituciones de la segunda mitad del siglo XX es su connotación manifiestamente pluralística: reconocen derechos provenientes de tradiciones ideológicas diferentes (por ejemplo, reconocen tanto los tradicionales derechos civiles y políticos de libertad, así como los derechos sociales), y reconocen también ulteriores bienes e intereses colectivos merecedores de ser perseguidos por los poderes públicos, todo ello sin establecer claras y definitivas relaciones de prioridad entre los derechos reconocidos.

Desde este punto de vista, hay una distancia evidente entre la realidad jurídica contemporánea y algunos influyentes modelos filosófico-políticos (pienso en particular en los de R. Nozick, J. RawLS, R. DwOrkin y J. HABERMAS) que conciben los dere- 
chos fundamentales como $a$ ) pocos; b) bien definidos, $c$ ) armónicos, estructurados de modo tal que no entran en conflicto entre ellos o, en cualquier caso, de modo que los eventuales conflictos son reconducidos a un marco que restituye una armonía general entre los derechos mismos; y sobre todo, $d$ ) prioritarios respecto de cualquier otra consideración que no sea a su vez traducible en términos de derechos individuales (la utilidad colectiva, el bien común, la gloria del Estado, el bien de la Patria, etc.) ${ }^{4}$. De manera muy sintética: en la filosofía política contemporánea la restricción del número de los derechos, o mejor dicho, de los intereses que pueden aspirar a la calificación de derechos, es funcional para evitar la necesidad de que los (pocos, verdaderos) derechos estén limitados y sean limitables en vista de consideraciones de otro tipo (utilidad colectiva, etc.). También es funcional a evitar que los derechos fundamentales sean relativizados y debilitados, ya sea porque están a la merced de continuas operaciones de mutuo ajuste con otros derechos o, principalmente, por exigencias heterogéneas 5 .

El panorama constitucional contemporáneo, como decía, exhibe características estructuralmente diferentes respecto de los desiderata de los modelos filosófico-políticos apenas mencionados: la multiplicidad y la heterogeneidad de los varios derechos fundamentales, y su igual dignidad constitucional son, probablemente, características inevitables en la constitución de una sociedad pluralista y democrática. Las constituciones democráticas contemporáneas: a) son fruto de compromisos entre diversas fuerzas políticas y expresan visiones diferentes de la sociedad $\left.{ }^{6} ; b\right)$ asumen el pluralismo, la diversidad de concepciones del bien en la sociedad no sólo como una cuestión ineliminable (esto es, como el modo en que de hecho están estructuradas las sociedades contemporáneas) sino también como un valor a preservar y, quizás, el único (meta-)

${ }^{4}$ Sobre la distancia conceptual entre los modelos filosófico-políticos citados en el texto y la práctica efectiva de los derechos fundamentales en los ordenamientos contemporáneos, J. WALDRON, «Security and Liberty: The Image of Balance», The Journal of Political Philosophy, vol. 11, 2003, pp. 191-210 (espec. pp. 196197); B. CELANO, «Diritti, principi e valori nello stato costituzionale di diritto: tre ipotesi di ricostruzione», Analisi e diritto, 2004, pp. 53-74.

5 En NozICK, la estrategia para eliminar el conflicto entre derechos consiste en restringir la categoría de los derechos a —-solamente- los derechos negativos (derechos de abstención por parte de un tercero), que pueden funcionar entonces como «side-constraints»; R. NozICK, Anarchia, stato e utopia (1974), Milano, il Saggiatore, 2005, pp. 246-247 [este punto es recogido con claridad por J. WALDRON, Rights in Conflict (1989), en Liberal Rights. Collected Papers 1981-1991, Cambridge, Cambridge U.P., 1993]. Una estrategia análoga es seguida por T. NAGEL, Questioni mortali (1979), Milano, il Saggiatore, 2001, pp. 113-114, y por M. IGNATIEFF, Una ragionevole apologia dei diritti umani (2001), Milano, Feltrinelli, 2003. También RAWLS se preocupa de individuar un elenco de pocas libertades fundamentales, que deben estar bien definidas, reconducibles a un esquema coherente; tales libertades tendrán «prioridad lexical» respecto a consideraciones de igualdad y justicia social [J. RaWLs, Una teoria della giustizia (1971), Milano, Feltrinelli, 1993, pp. 52, 66, 209-215, 255, 441 446; Id., Political Liberalism, New York, Columbia U.P., 1993, 2005³, pp. 291, 295, 296, 357]; merece la pena ver también la discusión crítica de H. L. A. HART, «Rawls on Liberty and Its Priority», University of Chicago Law Review, vol. 40, 1973, pp. 536-555. En la teoría de DwORKIN, responde a la misma exigencia de fondo la concepción de los derechos como trumps (triunfos) respecto a la justificación de decisiones políticas que persiguen finalidades colectivas: R. DwORKIN, «Rights as Trumps» (1981), en J. WALDRON (ed.), Theories of Rights, Oxford, Oxford U.P., 1984, pp. 153-167. En el mismo sentido, por último, vid. también J. HABERMAS, Fatti e norme. Contributi a una teoria discorsiva del diritto e della democrazia (1992), Milano, Guerini, 1996, cap. 6.

6 F. SCHAUER, «An Essay on Constitutional Language», UCLA Law Review, vol. 29, 1982, pp. 797-832, p. 806; G. SILVESTRI, «Linguaggio della costituzione e linguaggio giuridico: un rapporto complesso», Quaderni costituzionali, 1989 , pp. 229-255, p. 251; B. CELANO, «Come deve essere la disciplina costituzionale dei diritti??», en S. Pozzolo (a cura di), La legge e i diritti, Torino, Giappichelli, 2002, pp. 89-123; R. BIN, «Che cos'è la costituzione?», Quaderni costituzionali, 2007, 1, pp. 11-52 (especialmente pp. 22 y ss.). 
valor más importante que el resto ${ }^{7}$. En una sociedad fuertemente compacta, puede pensarse a modo de hipótesis, una constitución entendida como «tabla de los valores comunes» sería superflua; $c$ ) deben aplicarse, potencialmente, a toda la sociedad: una constitución semejante es casi literalmente un proyecto orgánico de fundación de un orden social $^{8}$, y $d$ ) están pensadas para durar mucho tiempo ${ }^{9}$.

En otras palabras, si una constitución está pensada para realizar estas tareas (como lo están, en general, las constituciones de las democracias pluralistas contemporáneas), entonces características como la multiplicidad de derechos, su formulación en modo no detallado, su conflictividad, su apertura a compromisos con consideraciones diferentes, etc., no representan un defecto, un mero efecto colateral, accidental e indeseado; lo que ocurre es que, simplemente, no podría ser de otra manera ${ }^{10}$. La única alternativa a este cuadro y a las operaciones interpretativas, en sentido amplio, sobre los derechos, que del cuadro se derivan, parece ser, sencillamente, no incluir en las constituciones un Bill of Rights, dejando la tarea de la determinación de los derechos fundamentales en manos de la dialéctica de la política regulada sólo por las reglas y los procedimientos de las democracias representativas ${ }^{11}$.

A sabiendas del hecho de que la pluralidad y la potencial conflictividad de los derechos fundamentales y de los principios constitucionales parece ser, en general, una característica aparentemente «estructural» del panorama constitucional contemporáneo, se han elaborado varios intentos, en la práctica de los tribunales y en el debate teórico y doctrinal, de limitar el ámbito y la efectiva incidencia del conflicto entre derechos ${ }^{12}$.

Un intento bastante común consiste en la distinción entra la (ilegítima) violación de un derecho, por un lado, y, por otro lado, su (inocua, o más bien necesaria) regulación ${ }^{13}$. Distinción perfectamente sensata, pero sujeta a una aclaración (cuestión que a

7 G. Postema, «In Defense of "French Nonsense". Fundamental Rights in Constitutional Jurisprudence», en N. MACCormicK y Z. BANKowski (eds.), Enlightenment, Rights and Revolution. Essays in Legal and Social Philosophy, Aberdeen, Aberdeen U.P., 1989, pp. 107-133 (especialmente pp. 125-126); G. ZAGREBELSKY, Il diritto mite. Legge diritti giustizia, Torino, Einaudi, 1992, pp. 16, 170-173; R. BIN, Che cos'è la Costituzione?, cit., pp. 22-25. Sobre el rol del pluralismo en el constitucionalismo contemporáneo, vid. también B. CELANO, «Ragione pubblica e ideologia», en I. TRujILlo y F. VIOLA (a cura di), Identità, diritti, ragione pubblica in Europa, Bologna, Il Mulino, 2007.

8 G. Zagrebelsky, La giustizia costituzionale, Bologna, Il Mulino, 1982, pp. 25-27, 125.

9 L. Gianformaggio, «Tempo della costituzione, tempo della consolidazione», Politica del diritto, 4, 1997, pp. 527-551; J. RAZ, «On the Authority and Interpretation of Constitutions. Some Preliminaries», en L. AleXander (ed.), Constitutionalism. Philosophical Foundations, Cambridge, Cambridge U.P., 2001, pp. 152-193.

10 «Que los principios incorporados por la Constitución sean incoherentes, o, mejor dicho, antitéticos, no es por tanto un defecto imputable a la Constitución, sino una característica estructural ineliminable de la misma» (R. Bin, Che cos'è la Costituzione?, cit., p. 23). Para una eficaz demostración de la irrazonabilidad e implausibilidad de una constitución que contenga pocos, armónicos y bien determinados derechos, $c f r$. B. Celano, Come deve essere la disciplina costituzionale dei diritti?, cit.

${ }_{11}$ Esta es, en efecto, la propuesta proveniente de los críticos de la judicial review of legislation: cfr. por ejemplo J. WALDRON, Law and Disagreement, Oxford, Oxford U.P., 1999; J. GOLDSWORTHY, The Sovereignty of Parliament. History and Philosophy, Oxford, Clarendon Press, 1999; A. Pintore, I diritti della democrazia, Roma-Bari, Laterza, 2003.

${ }_{12}$ Para una panorámica y una discusión de estas técnicas, vid. G. PINO, Diritti e interpretazione. Il ragionamento giuridico nello Stato costituzionale, cap. VI, Bologna, Il Mulino, 2010.

13 Sobre la distinción entre limitación/restricción (infringment) y violación/restricción (violation) de los derechos, cfr. A. GEWIRTH, «Are There Any Absolute Rights?» (1981), en J. WALDRON (ed.), Theories of Rights, 
menudo se da por implícita) acerca de bajo qué condiciones estamos en presencia de una violación o, por el contrario, de una regulación. A falta de argumentos explícitos en torno a qué diferencia la violación de la regulación de cierto derecho, la distinción resulta ser una mera petición de principio: los derechos en conflictos son considerados compatibles con un mero juego de palabras ${ }^{14}$.

Otra estrategia, bastante afín a la precedente, es la individuación de límites «implícitos» o «naturales» en los derechos ${ }^{15}$ : límites que, una vez individuados o, simplemente, «descubiertos», permiten establecer la coexistencia armónica entre derechos, distinguiendo aquello que «realmente» cae dentro del ámbito de aplicación de un derecho de aquello que, en cambio, sólo representa un uso aparente, o incluso abusivo, de un derecho. De este modo, definida, por ejemplo, la libertad de expresión como una libertad que, intrínsecamente, por su «naturaleza», no incluye ni la libertad de insultar ni la libertad de blasfemar, se evita in via definitoria el posible conflicto entre la libertad de expresión y la tutela de la personalidad y dignidad individuales, o también con la libertad religiosa.

Por último, otra posible estrategia es la individuación de jerarquías (axiológicas ${ }^{16}$ ) entre derechos fundamentales. De este modo, se distinguirá entre derechos fundamentales comunes y derechos fundamentales «supremos» ${ }^{17}$ (la doctrina del Tribunal Supremo norteamericano sobre preferred positions sigue la misma lógica) o entre derechos de varias «generaciones», atribuyendo a los derechos una importancia diferente con arreglo a la «generación» a la que pertenecen ${ }^{18}$. De modo que un eventual conflicto entre derechos de diversa importancia, o de diversa generación, será resoluble recurriendo a una suerte de orden lexicográfico preestablecido.

cit., pp. 91-109; R. NozICK, «Moral Complications and Moral Structures», Natural Law Forum, vol. 13, 1968, pp. 1-50 (espec. pp. 33-41); J. J. Thomson, «Some Ruminations on Rights», Arizona Law Review, vol. 19, 1977, pp. 45-60 (espec. pp. 47-48); R. Dworkin, Taking Rights Seriously, London, Duckworth, 19782, pp. 191-194, $197-200$

${ }_{14}$ Una aplicación de este tipo de razonamiento es la doctrina del «margen de valoración» desarrollada por el Tribunal Europeo de los Derechos del Hombre.

15 Para una discusión, $c f r$. L. PRIETO SANChís, «El constitucionalismo de los derechos», Revista Española de Derecho Constitucional, 24, 2004, pp. 47-72. Sobre la relación entre la retórica de los límites naturales de los derechos y el concepto de abuso del derecho, vid. G. PINO, «L'esercizio del diritto soggettivo e i suoi limiti. Note a margine della dottrina dell'abuso del diritto», Ragion pratica, 24, 2005, pp. 161-180.

${ }_{16}$ Una jerarquía axiológica es una relación entre dos normas, N1 y N2, tal que la norma N1 es considerada más importante respecto de la norma N2. La valoración de mayor importancia de N1 depende del hecho de que es considerada más adecuada a los valores, a los principios, a las doctrinas ético-políticas que inspiran el sistema jurídico, o un sub-sistema del mismo. GuAstini, Lezioni di teoria del diritto e dello Stato, Torino, Giappichelli, 2006; G. PINO, «Norme e gerarchie normative», Analisi e diritto, 2008.

${ }_{17}$ Cfr. Corte costituzionale n. 1146/1988. A propósito, M. TROPER, «La nozione di principio sovracostituzionale», Analisi e diritto, 1996, pp. 255-274.

${ }_{18}$ Según la presentación habitual, aunque no siempre reproducida en estos términos, la sucesión de las generaciones de los derechos sería la siguiente: derechos civiles (generación I), derechos políticos (generación II), derechos sociales (generación III), derechos de las generaciones futuras, y similares (generacón IV). Sobre el punto, vid. N. BobBIO, «Sui diritti sociali» (1996), en Teoria generale della politica, Torino, Einaudi, 1999, pp. 458-466; G. CoRSO, «Diritti umani», Ragion pratica, 7, 1996, pp. 59-67. Oportunamente R. BIN, «Diritti e fraintendimenti», Ragion pratica, 14, 2000, pp. 15-25, alerta contra los usos ideológicos de las generaciones de derechos, consistentes en sobreponer un orden de prioridad axiológica en lugar de lo que es una (aproximativa) sucesión cronológica. 


\section{LA POSICIÓN DE FERRAJOLI}

FERRAJOLI comparte con las posiciones teóricas y las estrategias doctrinales vistas en la sección precedente el objetivo de minimizar el ámbito y la incidencia del conflicto entre derechos, que tendría como efecto el debilitamiento y la relativización de los derechos ${ }^{19}$. La posición de FERRAJOLI, sin embargo, difiere de las apenas consideradas en lo siguiente: la de FERRAJOLI, en efecto, opera sobre un plano estrictamente conceptual y definitorio. FERRAJOLI trabaja, en otras palabras, sobre la definición formal, sobre la estructura de los derechos fundamentales y, sobre la base de este análisis, verifica (con tendencia a excluir) la posibilidad, así como el eventual alcance, de conflictos entre derechos fundamentales.

Comencemos con la definición general de qué es, según FERRAJOLI, un derecho fundamental, y con su distinción entre varias categorías de derechos fundamentales.

\subsection{La definición de derechos fundamentales}

FERRAJOLI propone una definición formal de derecho fundamental, en base a la cual «son "derechos fundamentales" todos aquellos derechos subjetivos que corresponden universalmente a "todos" los seres humanos dotados del status de personas, de ciudadanos o personas con capacidad de obrar; entendiendo por "derecho subjetivo" cualquier expectativa (de prestaciones) o negativa (de no sufrir lesiones) adscrita a un sujeto por una norma jurídica» ${ }^{20}$. Dada esta definición, la categoría de los derechos fundamentales se puede articular en ${ }^{21}$ :

a) Derechos (primarios) de libertad, los cuales, a su vez, se pueden presentar como:

$a^{1}$ ) Inmunidad de lesiones o constricciones («libertad de») 22; en esta categoría parecen estar incluidos todos aquellos derechos cuyo disfrute es (tendencialmente) coextensivo respecto a la titularidad misma: estos derechos no son, en sentido estricto, ejercidos $-\mathrm{o}$, mejor dicho, son ejercidos, simplemente, siendo titulares ${ }^{23}-$. Ejemplos de este tipo de derechos son los así denominados derechos de la personalidad (el derecho al nombre, el derecho a la propia imagen, el derecho a la intimidad, el derecho a la identidad personal), o el derecho a no ser torturado, la inviolabilidad del domicilio, el habeas corpus;

19 L. FerRajoli, Principia iuris, vol. 2, cit., p. 72.

${ }^{20}$ L. FERRAJOLI, Diritti fondamentali, cit., p. 5.

${ }^{21}$ Ibid.

22 Es necesario precisar que el uso del término «inmunidad» por parte de FERRAJOLI no guarda ninguna afinidad con el homólogo término difundido en la literatura teórica sobre los derechos de derivación hohfeldiana, ni con la noción hohfeldiana de «libertad». En términos hohfeldianos, aquello que FeRRAJOLI llama inmunidad sería calificable de «derecho en sentido estricto», o «pretensión» que tiene como correlativo un deber (negativo) de abstención. Cfr. W. N. HoHFELD, «Some Fundamental Legal Conceptions as Applied in Judicial Reasoning», Yale Law Journal, vol. 23, 1913, pp. 16 y ss.; G. PINO, «Diritti soggettivi. Elementi di un'analisi teorica», Materiali per una storia della cultura giuridica, 2, 2009.

23 A. PACE por ejemplo los llama «situaciones jurídicas subjetivas inactivas»: A. PACE, Problematica delle libertà costituzionali. Parte generale, Padova, Cedam, $2003^{3}$, pp. 95 y ss. 
$\left.a^{2}\right)$ Facultades de comportamientos no jurídicos igualmente inmunes a interferencias o constricciones («libertad de» además de «libertad frente a» —aunque por comodidad, en adelante, les denominaré, únicamente, como libertad «de»—) ${ }^{24}$; en esta categoría están incluidos los derechos cuyo ejercicio consiste en actividades concretas por parte del titular, «asistidas» por una obligación de abstención ajena de interferencias en la actividad del titular del derecho. Ejemplos de este tipo de derechos serían el derecho a la propiedad, la libertad de circulación, de asociación y de reunión, la libertad religiosa, la libertad de expresión.

b) Derechos sociales, es decir, derechos o prestaciones positivas (siempre que sean universales en el sentido anteriormente señalado): derecho a la educación, derecho a la salud (en los aspectos en que este derecho no es reconducible a la categoría a1: esencialmente, el derecho a recibir curas).

c) Derechos (secundarios) de autonomía, como los derechos civiles o los derechos políticos, esto es, todos aquellos derechos (o «derechos-poder») cuyo ejercicio produce efectos sobre las libertades positivas o negativas ajenas [entendidas estas últimas como meras libertades naturales, y no como derechos de libertad como los del tipo sub $a)^{25}$ ]; el ejercicio de los derechos secundarios de autonomía está sujeto a la ley, donde por «sujeción a la ley» hay que entender el conjunto de los límites y vínculos impuesto por los derechos de libertad y los derechos sociales.

Entre estos varios tipos de derechos fundamentales ${ }^{26}$ existe un orden jerárquico, con base al cual los derechos de la categoría c) (derechos de autonomía) están subordinados a aquellos de las categorías a) y b) (derechos de libertad y derechos sociales). En efecto, los derechos de autonomía sólo se pueden ejercer legítimamente en el ámbito del conjunto de los límites legales (legislativos y sobre todo constitucionales) constituido por los derechos de libertad y los derechos sociales. Esto es así porque los derechos de autonomía se ejercen produciendo actos jurídicos: a negocios jurídicos en el caso de la autonomía privada, actos legislativos en el caso de la autonomía pública. Y puesto que las normas que instituyen los otros derechos fundamentales normalmente son normas constitucionales ${ }^{27}$, los actos de ejercicio de los derechos fundamentales de autonomía están subordinados, por lo demás ${ }^{28}$, a los otros derechos fundamentales en virtud de la jerarquía de las fuentes del Derecho.

Los derechos fundamentales en conjunto (derechos de libertad, derechos sociales y derechos de autonomía), además, están por definición por encima de los dere-

${ }^{24}$ En términos hohfeldianos, derechos de este tipo consisten en una libertad (o tal vez un conjunto de libertades), junto a una pretensión que tiene por objeto un deber de abstención ajeno.

${ }^{25}$ En términos hohfeldianos, derechos de este tipo consisten en una libertad, eventualmente junto a un poder (en el caso en que el ejercicio del Derecho modifique posiciones jurídicas ajenas).

${ }^{26}$ Uno se pueda preguntar, por otra parte, si esta tipología es exhaustiva — si, en otras palabras, ¿da cuenta de todos los derechos que habitualmente consideramos fundamentales?: por ejemplo, ¿en qué categoría recae el derecho a la tutela judicial efectiva (derecho explícitamente calificado como inviolable por la constitución italiana, e incluso por el Tribunal constitucional en el conjunto de los principios constitucionales supremos?).

27 L. FerRAJOLI, I fondamenti dei diritti fondamentali, cit., pp. 293, 295, 330; Id., Principia iuris, vol. 2, cit., p. 74.

${ }^{28}$ En efecto, no está claro que los límites establecidos por una norma constitucional para el ejercicio de la autonomía pública o privada consistan sólo en derechos fundamentales: la constitución italiana, por ejemplo, individua también el «interés público» como límite a la iniciativa económica privada (además de a la seguridad, a la libertad y a la dignidad humana). 
chos patrimoniales: estos últimos pueden ser definidos como derechos no universales (mientras los derechos fundamentales son, por definición, adscritos «a todos»), disponibles, y tienen origen en un acto de naturaleza individual (negocios jurídicos, o actos administrativos) ${ }^{29}$.

\subsection{El ámbito de los conflictos entre derechos}

Con este marco teórico, la posibilidad de conflictos entre derechos fundamentales es — afirma FERRAJOLI- radicalmente reducida o, incluso, excluida. En efecto, en el análisis de FERRAJOLI:

$a^{1}$ Los derechos-inmunidad son intrínsecamente ilimitados y, por tanto, su garantía no interfiere con los otros derechos: estos derechos, afirma FERRAJOLI, «tienen tendencia a convivir sin recíprocas interferencias»; «los derechos de libertad [...] no entran, normalmente, en conflicto entre ellos» ${ }^{30}$; nótese que, incidentalmente, el uso, en los pasajes citados, de expresiones como «tendencia» y «normalmente» invita a pensar que para FERRAJOLI la no conflictividad de los derechos-inmunidad no es una necesidad lógica o conceptual, sino sólo una circunstancia empírica y, por tanto, contingente ${ }^{31}$.

$\left.a^{2}\right)$ Los derechos de libertad, entendidos como «libertad de», encuentran su límite en la imposición de convivencia con los derechos de libertad de los otros, con los cuales, efectivamente, pueden entrar en conflicto ${ }^{32}$, o incluso también con derechos fundamentales de otro tipo ${ }^{33}$; este es, aparentemente, el único caso de conflicto entre derechos fundamentales que FERRAJOLI está dispuesto a admitir, si bien prefiere traducir esta hipótesis al lenguaje de los «límites» de los derechos ${ }^{34}$.

b) Los derechos sociales no entran en conflicto con los derechos fundamentales de otro tipo, sino que más bien encuentran límites en los costes necesarios para asegurar su satisfacción: estos costes son satisfechos a través de la imposición fiscal y, por tanto, a través del sacrificio de los derechos patrimoniales (que son derechos subordinados a los fundamentales). Además, la elección de que los recursos vayan destinados a una prestación social o a otra es, según FERRAJOLI, una elección meramente política y no un límite impuesto a un derecho (social) por otro derecho ${ }^{35}$.

29 L. FerRajoli, Diritti fondamentali, cit., pp. 12-18; Id., Principia iuris, vol. 1, cit., pp. 759-765.

${ }^{30}$ Cfr. respectivamente I fondamenti dei diritti fondamentali, cit., p. 292; y Principia iuris, vol. 1, cit., p. 756. En ambos, la afirmación de los pasajes citados se refiere, textualmente, a todos los derechos primarios de libertad (es decir, tanto a la «libertad frente a» como a la «libertad de»). Sin embargo, como veremos inmediatamente, en otro lugar FERRAJOLI afirma exactamente lo contrario, es decir, que los derechos de «libertad de» pueden interferir con las libertades de los otros (I fondamenti dei diritti fondamentali, cit., p. 328; Principia iuris, vol. 1, cit., p. 757). Esto puede considerarse un lapsus calami (cfr. en efecto, Principia iuris, vol. 2, cit., p. 73, donde la afirmación se refiere exclusivamente a los derechos-inmunidad).

${ }_{31}$ Cfr. sin embargo Principia iuris, vol. 1, cit., p. 757, para la sugerencia de que las inmunidades no pueden «lógicamente» interferir con otros derechos. Para una defensa de la tesis lógica de que los derechos negativos no entran en conflicto, $c f r$. en cambio C. FRIED, Right and Wrong, Cambridge (MA), Harvard U.P., 1978, p. 110.

${ }_{32}$ L. Ferrajoli, I fondamenti dei diritti fondamentali, cit., pp. 328-330; Id., Principia iuris, vol. 1, cit., p. 757.

33 L. FerRajoli, Principia iuris, vol. 2, cit., p. 73.

34 Vid., por ejemplo, Principia iuris, vol. 1, cit., p. 757.

35 L. FERRAJOLI, I fondamenti dei diritti fondamentali, cit., p. 329; Id., Principia iuris, vol. 2, cit., p. 73. 
c) Los derechos de autonomía o derechos-poder están efectivamente destinados a «interferir y, por tanto, a entrar en conflicto con las esferas de otros sujetos» ${ }^{36}$; sin embargo, desde un punto de vista restringidamente jurídico, desde el enfoque de la nomodinámica, no pueden entrar en conflicto con (los otros) derechos fundamentales, en la medida en que están «constitutivamente» limitados por estos últimos. Tal y como hemos visto en un apartado anterior, el ejercicio de los derechos de autonomía está subordinado, en efecto, a las normas que regulan formas y contenidos de sus relativos actos de ejercicio, en garantía de los intereses públicos y de los derechos ajenos. Por tanto, en última instancia, «su relación con los otros derechos fundamentales no es configurable como "conflicto" sino como sujeción a la ley [...]; las leyes tienen la tarea de someter a límites, vínculos y controles jurisdiccionales» ${ }^{37}$ estos derechos. En definitiva, se podría dar un conflicto sólo «aparente» entre (actos de ejercicio de los) derechos de autonomía y otros derechos fundamentales, ya que se trataría de una antinomia susceptible de resolución (en favor de los segundos) recurriendo al criterio jerárquico.

Hasta aquí, la posición de FERRAJOLI. Que se trate, en sustancia, de una posición irenista o coherentista ${ }^{38}$ es sugerido, en el fondo, por el propio autor, a pesar de no compartir esta calificación. FERRAJOLI, en efecto, afirma que su intención es contrastar «la difundida tendencia en la filosofía jurídica actual a generalizar, enfatizar y dramatizar la existencia de los conflictos (entre derechos fundamentales)» ${ }^{39}$.

La posición de FERRAJOLI, por tanto, no es exclusivamente fruto de exigencias de pulcritud conceptual sino que también contiene inmediatas y declaradas consecuencias de política del Derecho, en cuanto está orientada a contrastar con la tendencia a atribuir fuerza expansiva a los derechos secundarios de autonomía (derechos-poder) en perjuicio de los otros derechos fundamentales ${ }^{40}$. Esta posición también influye, en el plano retórico, sobre la terminología empleada por FERRAJOLI, que prefiere eventualmente hablar de «límites» antes que de conflictos entre derechos, incluso en aquellos contextos en que admite explícitamente la plausibilidad de la tesis del conflicto $^{41}$ (sustitución terminológica que, en realidad, es recurrente en los autores que tienden a minimizar o a excluir la presencia de «genuinos» conflictos entre los derechos ${ }^{42}$ ).

36 L. FerRAJOLI, Principia iuris, vol. 1, cit., p. 756.

37 L. FerRajoli, I fondamenti dei diritti fondamentali, cit., p. 330 (de donde se ha extraído la cita); Id., Principia iuris, vol. 2, cit., pp. 74-75.

${ }_{38}$ Como hace notar, por ejemplo, A. PINTORE, Diritti insaziabili, en L. FerRajoli, Diritti fondamentali. Un dibattito teorico, cit., p. 184; la posición de FERRAJOLI es definida como «coherentista» por L. PRIETO SANCHís, «Constitucionalismo y garantismo», en M. CARBONELL y P. SALAZAR (coords.), Garantismo. Estudios sobre el pensamiento jurídico de L. Ferrajoli, cit., pp. $41-57$ (espec. pp. 46-47).

39 L. FerRAJOLI, Principia iuris, vol. 2, cit., p. 72.

${ }^{40}$ Ivi.

${ }^{41}$ Cfr., por ejemplo, L. Ferrajoli, I fondamenti dei diritti fondamentali, cit., pp. 328-330; Id., Principia iuris, vol. 1, cit., pp. 725-731.

42 Vid. una discusión de las estrategias retóricas y definitorias típicas de los planteamientos irenistas, y de las estrategias retóricas y teóricas a ellos asociados, en B. CELANO, Diritti, principi e valori nello stato costituzionale di diritto: tre ipotesi di ricostruzione, cit. 


\section{CRÍTICAS}

El modelo construido por FERRAJOLI, que espero haber reproducido correctamente, está expuesto, creo, a algunas observaciones críticas, conectadas entre sí. Intentaré exponerlas sometiendo a examen, a modo de confutación, varias posibles hipótesis en las que, para ser honestos, el fenómeno de la conflictividad entre derechos no parece en absoluto marginal.

Distinguiré, en particular, entre conflictos entre derechos fundamentales de diferente tipo, y conflictos entre derechos fundamentales del mismo tipo. A lo sumo, la restricción del ámbito de los conflictos entre derechos fundamentales llevada a cabo por FERRAJOLI parece proceder de la toma en consideración sólo de los posibles conflictos entre derechos de diferente tipo. En otras palabras, una vez individuadas las cuatro categorías de derechos fundamentales, FERRAJOLI sostiene que no se dan conflictos, o no se dan de manera significativa, entre derechos pertenecientes a categorías diferentes (los denominados conflictos inter-rights). De tal manera que, como hemos visto, FERRAJOLI tiende a excluir el conflicto:

a) Entre los derechos de «libertad frente a» $y$ los derechos de libertad «de» ${ }^{43}$ (porque las inmunidades son ilimitadas y «no se ejercen» en sentido propio, por lo que no entran en conflicto con otros derechos).

b) Entre derechos de libertad $y$ derechos sociales (se hablará sólo de opciones políticas en lo que respecta a las asignaciones de recursos disponibles).

c) Entre derechos de libertad y derechos sociales, por una parte, $y$ los derechos de autonomía, por otra parte: estos últimos, efectivamente, están subordinados a los primeros en virtud de la jerarquía de las fuentes del Derecho.

En primer lugar, intentaré mostrar que los conflictos entre derechos fundamentales del mismo tipo son posibles y frecuentes; y, en segundo lugar, que también entre los derechos fundamentales de diferente tipo se dan relaciones, y en definitiva conflictos, más articulados que los individuados por FERRAJOLI.

\subsection{Conflictos intra-rights}

Comencemos por los conflictos entre derechos fundamentales del mismo tipo (donde por «tipo» entiendo una de las cuatro grandes categorías de derechos fundamentales individuadas por FERRAJOLI). Conflictos de esta clase pueden, efectiva y frecuentemente, verificarse, e incluso pueden implicar varias instancias de un mismo tipo: por ejemplo, pueden darse conflictos no sólo entre dos derechos-inmunidad diferentes, sino también entre dos derechos-inmunidad del mismo tipo, imputables a dos o más sujetos diferentes (los denominados conflictos intra-rights) ${ }^{44}$. Éste es, analizado adecuadamente, un problema que se presenta para cualquier estrategia de eliminación

${ }^{43}$ Cfr. supra, nota 30 y texto correspondiente.

${ }^{4}$ Una crítica análoga, aunque no exactamente en los términos expuestos en el texto, en J. J. MoReso, «Sobre los conflictos entre derechos», en M. CARBOnell y P. SAlAZAR (coords.), Garantismo, cit., pp. 159170. Para una tipología articulada de los posibles conflictos entre derechos, F. KAMM, «Conflicts of Rights: 
del conflicto entre derechos a través de la instauración de algún tipo de jerarquía entre los mismos derechos.

Así pues, pueden verificarse posibles conflictos:

a) Entre derechos-inmunidad. Esta hipótesis de conflictos intra-rights es explícitamente excluida por FERRAJOLI ${ }^{45}$. La intuición de que los derechos de este tipo no entran en conflicto entre sí puede surgir de la circunstancia de que, como hemos visto, un derecho-inmunidad, en sentido propio, no se ejerce: el disfrute de una inmunidad exige, solamente, que no haya interferencias ajenas - puesto que el titular de un derecho-inmunidad no hace literalmente nada, sino que se limita, por así decir, a tener un derecho, parece realmente extraño que una inmunidad pueda entrar en conflicto con otros derechos, y sobre todo con otras inmunidades. Sin embargo, se trata de una intuición engañosa. Piénsese en los siguientes ejemplos: el conflicto entre el derecho a la vida de Ticio y el derecho a la vida de Caio (como ejemplo, en hipótesis, de legítima defensa o de estado de necesidad); o el conflicto entre el derecho de Caio a no ser torturado (para obtener informaciones sobre un probable atentado terrorista a un avión), y el derecho a la vida de terceros (por ejemplo, de los pasajeros del avión sobre el que pesa la amenaza de atentado) ${ }^{46}$; o el conflicto entre el derecho a la salud (entendido como derecho-inmunidad y no como derecho social) de Ticio y el análogo derecho a la salud de Caio (como cuando se somete a alguien a vacuna obligatoria, con riesgo de lesiones para su salud, para tutelar la salud de la colectividad ${ }^{47}$ ); o, finalmente, el conflicto entre el derecho al nombre de Ticio y el derecho al nombre de Caio (cuando hay controversia acerca del uso legítimo de un determinado nombre).

Parecería, entonces, que los derechos-inmunidad pueden entrar en conflicto entre ellos. En otras palabras, si un derecho-inmunidad consiste en la ausencia de lesiones de los intereses protegidos por aquel derecho-inmunidad, nada impide, conceptualmente, que la lesión esté determinada por la exigencia de tutelar otro derecho-inmunidad (esto es, por la garantía secundaria de otro derecho-inmunidad), o incluso por el mero ejercicio de otro derecho-inmunidad (esto es, por su propia garantía primaria).

b) Entre los derechos de «libertad de». Esta hipótesis también es tomada en consideración por FERRAJOLI, aunque en este caso reconoce abiertamente la posibilidad de conflictos entre derechos. Sin embargo, según FERRAJOLI se trata de conflictos normalmente ya («a monte») resueltos por el mismo Derecho positivo, por las normas constitucionales que atribuyen el derecho fundamental: por tanto, para gestionar estos conflictos no son necesarios ulteriores intervenciones en sede jurisdiccional o, en cualquier caso, interpretativa ${ }^{48}$. Por desgracia, los ejemplos aportados por FERRAJOLI

\footnotetext{
Typology, Methodology and Non-Consequentialism», Legal Theory, vol. 7, 2001, pp. 239-255; G. Pino, Diritti e interpretazione, cit., cap. VI.

45 Vid. supra, notas 30-31 y texto correspondiente.

46 Un caso afín ha sido resuelto por el Tribunal constitucional federal alemán, a propósito de la legitimidad constitucional de una ley que habría permitido a las fuerzas militares aéreas alemanas abatir un avión civil que hubiera sido objeto de desviación terrorista, para evitar que el avión fuera a su vez utilizado como arma contra otros civiles. El Tribunal ha sostenido que una ley semejante violaba el principio supremo de la tutela de la dignidad (de los pasajeros del avión desviado). Cfr. Bundesverfassungsgericht, Sentencia de 15 de febrero de 2006, 1 BvR 357/05.

47 Corte costituzionale n. 258/1994.

${ }^{48}$ L. Ferrajoli, Principia iuris, vol. 2, cit., p. 73.
} 
para corroborar esta afirmación (el art. 21 para la relación entre libertad de expresión y derecho al honor; el art. 40 para la relación entre derecho a la huelga y otros derechos) terminan por resultar un poco apodícticos: que el art. 21 de la constitución italiana resuelva explícitamente el conflicto entre la libertad de expresión y el derecho al honor y a la reputación (limitando la primera en favor del segundo) es banalmente desmentido por la lectura del mismo artículo. En realidad este conflicto no es resuelto por la Constitución; que el art. 40 de la constitución diga que el derecho a la huelga se ejerce en el ámbito de las leyes que lo regulan no resuelve el conflicto entre el derecho a la huelga y otros derechos (por ejemplo la libertad de circulación de los ciudadanos, o en general los derechos de los usuarios de los servicios públicos), sino que exige la solución al legislador — quien, por otra parte, puede no intervenir, o intervenir de modo lagunoso y fragmentario, como efectivamente ha ocurrido en Italia, o incluso puede intervenir de manera irrazonable, limitando excesivamente el derecho a la huelga en favor de otros intereses-. En definitiva, está por demostrar que sea la constitución lo que realmente resuelve estos conflictos.

Además, puesto que los derechos fundamentales son habitualmente proclamados en términos amplios, genéricos, indeterminados, e incluso los eventuales límites expresados son formulados del mismo modo, la suposición de que todas las hipótesis de conflicto entre un derecho y un bien contrapuesto estén predeterminados y resueltos ya de antemano parece decididamente optimista (el hecho que según la constitución italiana las buenas costumbre sean un límite a la libertad de expresión es sólo el inicio de un recorrido argumentativo que incluye valoraciones de razonabilidad y proporcionalidad, ponderaciones, etc.).

c) Entre derechos sociales. Piénsese en el caso en que se debe decidir si destinar los recursos disponibles a un cierto tipo de prestación social en perjuicio de otra: destinar todos los recursos disponibles a la tutela del derecho a la salud puede perjudicar la efectividad del derecho a la educación (y viceversa). FERRAJOLI, tal y como hemos visto, considera esta hipótesis no como un conflicto entre derechos sino como un problema de elecciones de carácter meramente político. No obstante, no estoy seguro de que esta afirmación pueda ser fácilmente conciliada con la tesis de FERRAJOLI según la cual la falta de garantía de un derecho social es una verdadera (e indebida) laguna en sentido propio, y no una mera omisión política ${ }^{49}$. De ser esto así, no se entiende por qué una disciplina legislativa asimétrica, por así decir, de derechos sociales, todos previstos en la Constitución, no puede ser una antinomia, y por tanto un conflicto entre derechos (sociales), sino sólo una mera elección política, mientras una eventual decisión de los poderes públicos de no prestar alguna garantía para ambos derechos sociales sería, en cambio, una «laguna indebida».

d) Entre derechos de autonomía. Los derechos de autonomía se ejercen produciendo actos jurídicos, por lo que, por lo menos algunos posibles conflictos entre derechos de este tipo, podrán ser resueltos con base en la jerarquía de las fuentes del Derecho vigente en un ordenamiento dado. Habría que concluir, por tanto, que, de

${ }^{49}$ L. Ferrajoli, Diritti fondamentali, cit., pp. 30-31; Id., Principia iuris, vol. 2, cit., pp. 76, 400-401. Ulteriores críticas sobre el excesivo relieve de la elección política, en perjuicio de la dimensión jurídica, de los derechos sociales, en L. PRIETO SANCHÍs, «Principia iuris: una teoría del Derecho no (neo)constitucionalista para el Estado constitucional», Doxa, vol. 31, 2008, pp. 325-354. 
manera tendencial, la autonomía pública prevaldrá siempre sobre la autonomía privada (la ley prevale siempre sobre los contratos, por ejemplo).

\subsection{Conflictos inter-rights}

FERRAJOLI admite expresamente la posibilidad de algunos conflictos entre derechos de diverso tipo (donde por «tipo», nuevamente, entenderé una de las cuatro grandes categorías de derechos fundamentales individuadas por FERRAJOLI), si bien tal posibilidad, tras el debido análisis teórico, queda muy reducida: los conflictos o no se dan, o se dan pero son irrelevantes. En realidad, creo que la posibilidad de conflictos entre derechos fundamentales es bastante más amplia de lo que admite FERRAJOLI. En particular, desarrollando una combinación de posibilidades a partir de la taxonomía diseñada por FERRAJOLI, podrían darse:

a) Conflictos entre derechos de libertad (libertad «de») y derechos-inmunidad (libertad «frente a»). FERRAJOLI, como hemos visto, afirma que mientras las inmunidades son ilimitadas, las libertades «de» encuentran varios límites, entre los cuales el mismo respeto de las inmunidades ${ }^{50}$. Ahora bien, no me parece que este modo de plantear la cuestión sea del todo convincente. De hecho, el ámbito (jurídico) de extensión de las inmunidades no es nunca ilimitado sino que es exactamente el resultado de una ponderación entre los intereses que justifican la atribución de una inmunidad y los intereses que justifican los derechos concurrentes ${ }^{51}$.

Piénsese en los ejemplos utilizados por FERRAJOLI en varias ocasiones del derecho a la intimidad, o del derecho al honor, en relación con la libertad de expresión: por un lado, es cierto que la libertad de expresión (que es una libertad «de») está limitada por el derecho al honor y el derecho a la intimidad ajenos (que son libertades «para»). Pero, por otro lado, es exactamente igual de cierto lo contrario ${ }^{52}$, porque la extensión del ámbito de tutela jurídicamente reconocido a la intimidad y al honor será individuada con arreglo al valor (importancia) de la libertad de expresión. De esta manera, se puede sostener que, en efecto, en algunos casos es oportuna o incluso necesaria una compresión de los derechos-inmunidad al honor y a la intimidad, según la importancia de ciertas formas de expresión (la intimidad de los cargos políticos, por ejemplo, en relación con el derecho de información). Y que a una inmunidad, lejos de ser ilimitada, se le puedan poner límites en razón de otros derechos que van en dirección opuesta, es verdad incluso cuando se hace referencia a otros derechos fundamentales del mismo titular (en el estado social, el acceso a muchos derechos sociales está subordinado a

50 L. Ferrajoli, Principia iuris, vol. 1, cit., p. 757.

51 Para la adhesión de FERRAJOLI a la teoría del interés como justificación de los derechos subjetivos (sostenido en la teoría del Derecho contemporánea por ejemplo por J. RAZ, N. MACCORMICK, J. WALDRON y M. Kramer), cfr. Principia iuris, vol. 1, cit., pp. 641-643. Para una sintética presentación de esta teoría G. PINO, Diritti soggettivi. Elementi di un'analisi teorica, cit.

52 Y de otro lugar es la afirmación, un poco curiosa, de que las inmunidades no entran en conflicto nunca con los derechos de libertad, mientras que, en cambio, los derechos de libertad pueden entrar en conflicto con las inmunidades — posición que recuerda a aquella que A. MARMOR llama concepción «newtoniana» de los derechos (la idea según la cual cada derecho se mueve libremente en un espacio moral vacio, hasta que no se encuentra límites externos derivados de la colisión con otro derecho)—: A. MARMOR, «On the Limits of Rights», Law and Philosopby, vol. 16, 1997, pp. 1-18 (p. 7). 
la comunicación de diferentes informaciones personales del titular del derecho a la administración pública: por tanto, derecho social a una prestación vs. derecho a la intimidad), o de otros titulares (a modo de hipótesis, e incluso frecuentemente admitidas, estarían las limitaciones del derecho a la intimidad por exigencias ajenas de tutela de la salud).

Por tanto, las inmunidades no son en absoluto ilimitadas: su perímetro es el resultado de una ponderación (no importa en este momento si es hecha de manera estable por el legislador o si es hecha caso por caso por la jurisprudencia) entre el interés que justifica la atribución de la inmunidad y los intereses que justifican otros derechos (inmunidad, libertad «de», o «derechos sociales»). Y la ponderación es necesaria porque las inmunidades pueden entrar en conflicto con los otros derechos.

b) Conflictos entre derechos-inmunidad («libertad frente a») y derechos de «libertad de» por una parte, y derechos sociales, por la otra. En primera instancia, FERRAJOLI sostiene que no se trata de verdaderos conflictos, sino de cuestiones políticas acerca de la distribución de recursos disponibles. Sin embargo, el mismo FERRAJOLI da un buen ejemplo de conflicto de este tipo: entre el derecho a la huelga de los empleados de hospital («libertad de») y el derecho a la salud (derecho social) ${ }^{53}$; no queda claro, no obstante, cómo este tipo de conflicto es tratado en el ámbito de su teoría, más allá de una genérica referencia al marco de los límites legales (legislativos y constitucionales) de los derechos de libertad. Nótese que precisamente este es el problema: los límites legales de la libertad podrían no estar establecidos, o podrían estar establecidos de manera fragmentaria, indeterminada y lagunosa, o de manera tal que se restringiera excesivamente el derecho de libertad. Recuérdese que el mismo poder legislativo (esto es, las fuentes de los «límites legales» de la libertad) está, a su vez, subordinado al respeto de los derechos fundamentales ${ }^{54}$ : se desata así un potencial círculo vicioso entre el Derecho y su límite.

c) Conflicto entre derechos-inmunidad («libertad frente a») y derechos de «libertad de» por un lado, y derechos de autonomía, por el otro. El conflicto entre estos derechos es resuelto, tal y como hemos visto, haciendo referencia a la jerarquía de las fuentes del Derecho. El punto débil de este planteamiento es que la rígida jerarquía entre derechos de autonomía y derechos de libertad, consecuencia del diferente grado jerárquico entre las normas (negociales y legislativas) producidas en el ejercicio de los primeros y las normas constitucionales que instituyen los segundos, no da cuenta de algunas intuiciones no controvertidas sobre el modo en que funcionan los derechos fundamentales (a decir verdad, se sigue la total ilegitimidad). Se pueden imaginar limitaciones (en apariencia del todo razonables y sensatas, y - en un punto de Derecho positivo- legítimas) de la libertad de expresión en el ámbito de una relación contractual de trabajo (piénsese en el deber de discreción de un trabajador dependiente o empleado, o en la obligación de respetar la línea editorial de un periódico por parte de un periodista) o, también dentro del ámbito de la relación de trabajo, en los límites a la libertad de iniciativa económica del trabajador dependiente o empleado ${ }^{55}$.

53 Principia iuris, vol. 2, cit., pp. 72 y 73.

54 Cfr. supra, nota 25 y texto correspondiente.

55 Por ejemplo, el Derecho italiano prevé el deber de fidelidad del trabajador y los denominados pactos de no concurrencia incluso más allá de la finalización de la relación de trabajo: arts. 2105 y 2125 CC. Para una 
A propósito de los derechos de autonomía, FERRAJOLI admite que los derechos de autonomía están «destinados a entrar en conflicto [con las libertades positivas y negativas] en el caso de que no sean jurídicamente limitados y disciplinados» ${ }^{56}$. El conflicto debería ser, por tanto, excluido en virtud del hecho de que en el estado constitucional de Derecho estos límites jurídicos existen y son, precisamente, los derechos de libertad y los derechos sociales. Ahora bien, aun concediendo que las cosas sean así, los derechos de autonomía no darían lugar a conflictos sólo si sus límites jurídicos (legislativos, constitucionales) son adecuadamente precisos y puntillosos, sino que cada vez que la regulación jurídica «superior» (los derechos de libertad y los derechos sociales) sea suficientemente vaga, imprecisa, lagunosa o antinómica, entonces aparecen de nuevo las posibilidades de que surjan conflictos.

Se puede sostener que, por un lado, la dejadez de los legisladores de carne y hueso (lejanos del ideal ilustrado del legislador perfectamente racional) y, por otro lado, la inevitable complejidad y antinomicidad de la dimensión ética sustancial de los ordenamientos jurídicos constitucionales contemporáneos ${ }^{57}$, alejan bastante a este modelo de la realidad.

\section{EL PROBLEMA DE FONDO}

El modelo teórico diseñado por FERRAJOLI, así pues, neutraliza de manera sólo aparente el problema de los conflictos entre derechos fundamentales, mientras que en realidad termina por admitir —al menos implícitamente — la inevitabilidad de los mismos. Ahora bien, creo que las críticas que he intentado argumentar hasta aquí, aun admitiendo que den en el blanco, tienen en el fondo un hilo común, es decir, son reconducibles a un único tipo de problema. Me explicaré.

La estrategia de reducción de FERRAJOLI, cuando no de exclusión, de los conflictos entre derechos fundamentales se basa, tal y como hemos visto, en la neta distinción entre tipos de derechos: algunos de estos derechos, añade FERRAJOLI, no pueden entrar en conflicto por, digámoslo así, razones conceptuales; otros por razones de jerarquía de las fuentes (la nomodinámica). El éxito de la estrategia depende, entonces, de la capacidad de defensa que tenga la tesis de la neta distinción entre tipos de derechos.

Creo que es aquí donde realmente está el problema. FERRAJOLI observa, creo que de manera nada controvertida, que los derechos fundamentales son posiciones moleculares, complejas, que contienen tanto elementos «positivos» como «negativos» ${ }^{58}$. De

\footnotetext{
discusión análoga sobre el punto, M. JoRI, «Aporie e problemi nella teoria dei diritti fondamentali», en L. FERRAJOLI, Diritti fondamentali. Un dibattito teorico, cit.

56 L. FerRAJOli, I fondamenti dei diritti fondamentali, cit., p. 292; Id., Principia iuris, vol. 1, cit., p. 756.

57 Vid. B. Celano, Come deve essere la disciplina costituzionale dei diritti?, cit.

58 L. Ferrajoli, Principia iuris, vol. 1, cit., pp. 325, 327, 643; vol. 2, pp. 67-68, 398. Sobre la idea de los derechos como posiciones «moleculares», vid. ya W. HoHFELD, Some Fundamental Legal Conceptions as Applied in Judicial Reasoning, cit.; cfr. además J. FINNIS, Natural Law and Natural Rights, Oxford, Clarendon Press, 1980, p. 201; J. WALDRON, «Introduction», en J. WALDRON (ed.), Theories of Rights, cit., pp. 10-11; G. Postema, In Defense of «French Nonsense», cit., pp. 109-114; J. J. ThOMson, The Realm of Rights, Cambridge (MA), Harvard U.P., 1990, pp. 55 y ss. (algunos derechos son cluster-rights, «racimos» de posiciones hohfeldianas, «rights that contain other rights»); M. BARBERIS, «I conflitti fra diritti tra monismo e pluralismo etico»,
} 
esta observación derivan, no obstante, dos implicaciones importantes. La primera es que si un derecho es un agregado molecular de posiciones subjetivas, esto no significa que todas estas posiciones subjetivas tengan la misma importancia (¿importancia respecto de qué? Respecto de los intereses que justifican la atribución del Derecho). Al contrario, es bastante plausible que del derecho fundamental surjan varias olas de específicas posiciones subjetivas o, en otros términos, que son posibles varias modalidades de ejercicio de un mismo derecho fundamental, no todos igualmente importantes respecto de los intereses que el derecho intenta proteger ${ }^{59}$. Una reconstrucción similar, en línea con la denominada teoría dinámica de los derechos ${ }^{60}$ (que normalmente son desarrollos de la teoría del interés, compartida por FERRAJOLI) explica perfectamente la hipótesis, vista un poco más arriba, según la cual un derecho (primario) de libertad puede ceder frente a un derecho (secundario) de autonomía, o bien la hipótesis según la cual un derecho fundamental (en teoría indisponible) puede ser objeto de actos de disposición, «degradándose» así a derecho patrimonial ${ }^{61}$.

La segunda implicación es que raramente un determinado derecho fundamental es exclusivamente un derecho de libertad «de», o de libertad «para», o un derecho social, o un derecho de autonomía: más frecuentemente, siendo como es un agregado molecular, un derecho fundamental es muchas de (o todas) estas cosas a la vez. El ejemplo típico es el derecho a la salud, que es al mismo tiempo un derecho-inmunidad (como expectativa de que la propia integridad psico-física no sufra lesiones), y un derecho social (como expectativa de recibir prestaciones sanitarias) ${ }^{62}$; si por último, también se incluye en el derecho a la salud el aspecto de la autodeterminación sanitaria ${ }^{63}$, el derecho a la salud se presenta también como libertad «de». Otro ejemplo es el derecho a la tutela judicial efectiva: en ciertos aspectos parece ser una libertad «de» porque incluye la libertad de cumplir una vasta y heterogénea serie de comportamientos concernientes

Analisi e diritto, 2005; Id., Etica per giuristi, Roma-Bari, Laterza, 2006, cap. I (distinción entre micro-derechos y macro-derechos).

${ }_{59}$ Cfr. J. WaLdRON, Rights in Conflict, cit., pp. 212, 215, 218. Cfr. también R. BIN, Diritti e fraintendimenti, cit., p. 17: «la definición del "Derecho" no es como una olla, respecto a la cual un determinado fenómeno está dentro o fuera, tertium non datur. Más bien se comporta como un paraguas durante un fuerte chubasco: hay un punto en que la protección es máxima, y después, a medida que el chubasco se aleja, la protección es menos eficiente; es difícil decir en qué punto se está totalmente fuera del paraguas, también porque en buena parte depende del viento».

60 Para algunos ejemplos de esta posición, con matices diferentes: J. RAZ, «Legal Rights» (1984), en Ethics in the Public Domain. Essays in the Morality of Law and Politics, Oxford, Oxford U.P., 1994, pp. 254-276; N. MACCORMICK, «Rights in Legislation», en P. M. S. HACKeR y J. RAz (eds.), Law, Morality, and Society. Essays in Honour of H. L. A. Hart, Oxford, Clarendon Press, 1977, pp. 198-209.; J. WALDRON, Rights in Conflict, cit., espec. pp. 212-214; Id., The Right to Private Property, Oxford, Clarendon Press, 1988, pp. 84 y ss.; M. Kramer, «Rights Without Trimmings», en M. KRAmer, N. Simmonds y H. SteIner, A Debate over Rights. Philosophical Enquiries, Oxford, Oxford U.P., 1998, pp. 7-111 (espec. pp. 41-44); A. PINTORE, Diritti insaziabili, cit., espec. pp. 192 a 199. Vid. una discusión exhaustiva en B. CELANO, «I diritti nella jurisprudence anglosassone contemporanea», Analisi e diritto, 2001, pp. 1-58 (espec. pp. 41-50).

${ }_{61}$ Piénsese en las siguientes hipótesis: un contrato para el disfrute comercial de la imagen (el denominado right of publicity); el consenso en el tratamiento de datos personales; una entrevista sobre hechos privados (actos de disposición del derecho a la intimidad). En general sobre estos argumentos, V. ZeNo-ZenCOVICH, «Limitazioni contrattuali alla manifestazione del pensiero», Il diritto dell'informazione e dell' informatica, 1995, pp. 991-1001; G. RESTA, Autonomia privata e diritti della personalità, Napoli, Jovene, 2005.

62 Corte costituzionale n. 445/1990.

${ }^{63}$ Cfr. Corte costituzionale n. 438/2008. 
a la defensa en el juicio, libres de (indebidas) interferencias ${ }^{64}$; pero, en otros aspectos, parece ser un derecho social, porque presupone una serie de prestaciones positivas, entre las cuales la asistencia gratuita y la defensa de oficio, un servicio de traducción en el caso de que el imputado no hable la lengua en la cual se le acusa y, naturalmente, la existencia de un juez imparcial ${ }^{65}$.

FERRAJOLI, finalmente, admite que todos los derechos fundamentales (y no sólo los sociales) tienen un coste sobre el erario público (además, puede pensarse, de que lo tenga sobre terceros); no queda claro cómo esta observación, en absoluto controvertida, liga con la idea de que algunos derechos fundamentales son ilimitados mientras que otros encuentran límites sólo en derechos fundamentes ajenos (y en cambio nunca en la existencia de recursos materiales).

En conclusión: si es verdad que algunos derechos fundamentales entran efectivamente en conflicto (como FERRAJOLI admite); si es verdad, además, que las hipótesis de conflicto están probablemente más extendidas que aquéllas que FERRAJOLI está dispuesto a reconocer (como he intentado mostrar); y si es verdad, por último, que muchos o quizás todos los derechos fundamentales, en cuanto agregados moleculares complejos de posiciones subjetivas, pertenecen transversalmente a más de una de las categorías teóricas individuadas por FERRAJOLI (como igualmente FERRAJOLI admite); si todo lo anterior es verdad, considero confirmada, en definitiva, la tesis de que los conflictos entre derechos fundamentales son, de hecho, inevitables.

\section{(Traducción de Pau Luque Sánchez)}

${ }^{64}$ Nótese, sin embargo, que para FERRAJOLI los comportamientos que constituyen ejercicio de una libertad «de» son no jurídicos (vid. supra, $\$ 2.1$ ); por el contrario, la gran parte de los actos de ejercicio del derecho a la tutela judicial efectiva está constituida por actos jurídicos (comparecer en juicio, nombrar a un abogado, y después el desarrollo de toda una serie de actos procesales por parte del defensor). Esto complica (como decía supra, n. 27) la tarea de enmarcar el derecho a la tutela judicial efectiva en las categorías propuesta por FERRAJOLI.

${ }^{65}$ Por otra parte, no queda claro si todas estas deben ser consideradas garantías primarias o bien secundarias del derecho a la tutela judicial efectiva. 